\title{
Level sequence and splitting identification of closely-spaced energy levels by angle-resolved analysis of the fluorescence light
}

\author{
Z. W. Wu, ${ }^{1,2}$ A. V. Volotka, ${ }^{1,3}$ A. Surzhykov, ${ }^{4,5}$ C. Z. Dong, ${ }^{2}$ and S. Fritzsche ${ }^{1,6}$ \\ ${ }^{1}$ Helmholtz-Institut Jena, Fröbelstieg 3, D-07r43 Jena, Germany \\ ${ }^{2}$ Key Laboratory of Atomic and Molecular Physics \& Functional Materials of Gansu Province, \\ College of Physics and Electronic Engineering, Northwest Normal University, Lanzhou 730070, P.R. China \\ ${ }^{3}$ Department of Physics, St. Petersburg State University, 198504 St. Petersburg, Russia \\ ${ }^{4}$ Physikalisch-Technische Bundesanstalt, Bundesallee 100, D-38116 Braunschweig, Germany \\ ${ }^{5}$ Technische Universität Braunschweig, D-38106 Braunschweig, Germany \\ ${ }^{6}$ Theoretisch-Physikalisches Institut, Friedrich-Schiller-Universität Jena, Max-Wien-Platz 1, D-07743 Jena, Germany
}

(Dated: July 31, 2018)

\begin{abstract}
The angular distribution and linear polarization of the fluorescence light following the resonant photoexcitation is investigated within the framework of the density matrix and second-order perturbation theory. Emphasis has been placed on "signatures" for determining the level sequence and splitting of intermediate (partially) overlapping resonances, if analyzed as a function of the photon energy of the incident light. Detailed computations within the multiconfiguration Dirac-Fock method have been performed especially for the $1 s^{2} 2 s^{2} 2 p^{6} 3 s \quad J_{i}=1 / 2+\gamma_{1} \rightarrow\left(1 s^{2} 2 s 2 p^{6} 3 s\right)_{1} 3 p_{3 / 2} J=$ $1 / 2,3 / 2 \rightarrow 1 s^{2} 2 s^{2} 2 p^{6} 3 s \quad J_{f}=1 / 2+\gamma_{2}$ photoexcitation and subsequent fluorescence emission of atomic sodium. A remarkably strong dependence of the angular distribution and linear polarization of the $\gamma_{2}$ fluorescence emission is found upon the level sequence and splitting of the intermediate $\left(1 s^{2} 2 s 2 p^{6} 3 s\right)_{1} 3 p_{3 / 2} \quad J=1 / 2,3 / 2$ overlapping resonances owing to their finite lifetime (linewidth). We therefore suggest that accurate measurements of the angular distribution and linear polarization might help identify the sequence and small splittings of closely-spaced energy levels, even if they can not be spectroscopically resolved.
\end{abstract}

PACS numbers: $31.10 .+\mathrm{z}, 31.15 . \mathrm{aj}$

\section{INTRODUCTION}

In atoms and ions with complex shell structures, levels are often closely spaced in energy and, thus, difficult to resolve spectroscopically. Up to the present, therefore, suitable spectroscopic schemes for resolving the level structure have played important role in studying the structure of atomic systems [1-3]. Experimentally, indeed, great effort has been made to improve the resolution of photon detectors [4 [6] and to obtain ever detailed spectral information. However, when the level splitting becomes comparable with the (natural) width of the transitions, it becomes inherently difficult to resolve both, the sequence as well as the splitting of the energy levels owing to their (partial) overlap, even if high-resolution spectroscopy is applied. In this case, an alternative route to identify the sequence and splitting of energy levels becomes highly desirable.

In the past decades, much emphasis in atomic spectroscopy has been placed upon the angle-resolved properties of emitted light, such as the angular distribution and linear polarization [7, 8]. When compared to the total cross sections and decay rates for the photon emission from atoms and ions, angle-resolved measurements were found more sensitive with regard to the details in the electron-electron and electron-photon interactions. For example, the angular distribution and linear polarization of fluorescence emission were discussed in studying the Breit interaction in dielectronic recombination processes [9 12], the hyperfine interaction [13, 14] in electron-atom collisions as well as the multipole mixing in the interaction of ions with the radiation field [15, 16].

However, less attention has been paid to the influence of overlapping resonances upon the atomic fluorescence, in contrast to the autoionization of inner-shell excited atoms 17-19. Only rather recently, we explored the angular and polarization properties of the emitted photons in the two-step radiative cascade $1 s 2 p^{2} \quad J_{i}=1 / 2,3 / 2 \rightarrow$ $1 s 2 s 2 p J=1 / 2,3 / 2+\gamma_{1} \rightarrow 1 s^{2} 2 s J_{f}=1 / 2+\gamma_{1}+\gamma_{2}$ of lithium-like tungsten, which proceeds via such overlapping intermediate resonances [20, 21]. While, for an initially aligned $1 s 2 p^{2} \quad J_{i}=3 / 2$ level, a remarkably strong dependence was obtained for the second-step fluorescence photons upon the splitting of the two (overlapping) $1 s 2 s 2 p \quad J=1 / 2,3 / 2$ resonances, no effect was found with regard to the sequence of these resonances due to the mutual cancelation of the sequence-dependent summation terms. In this work, we therefore study the process of resonant photoexcitation and subsequent fluorescence of atoms to better understand how both, the level sequence and splitting can be made visible for closelyspaced energy levels. To this end, second-order perturbation theory and the density matrix formalism are employed in order to derive and analyze general expressions for the angular distribution and linear polarization of the emitted radiation. Though these expressions are applicable to many-electron atoms (or ions), and are independent of their particular shell structure, we shall consider below the $2 s \rightarrow 3 p$ inner-shell photoexcitation and subsequent fluorescence emissions of sodium atom, e.g., 
$1 s^{2} 2 s^{2} 2 p^{6} 3 s \quad J_{i}=1 / 2+\gamma_{1} \rightarrow\left(1 s^{2} 2 s 2 p^{6} 3 s\right)_{1} 3 p_{3 / 2} \quad J=$ $1 / 2,3 / 2 \rightarrow 1 s^{2} 2 s^{2} 2 p^{6} 3 s \quad J_{f}=1 / 2+\gamma_{2}$. For inner-shell excited sodium, the $\left(1 s^{2} 2 s 2 p^{6} 3 s\right)_{1} 3 p_{3 / 2} \quad J=1 / 2,3 / 2$ resonances are well isolated from other (fine-structure) levels of the $2 s \rightarrow 3 p$ excitation and their level splitting is comparable to the (natural) widths in the excitation and decay of these resonances.

This paper is structured as follows. In the next section, we present general expressions for the (secondorder) transition amplitude of the photoexcitation and associated radiative decay of the atoms (or ions). This transition amplitude is then employed to express the angular distribution and linear polarization of the emitted fluorescence photons. In Sec. III. we apply these expressions particularly to the $2 s \rightarrow 3 p$ photoexcitation of the $2 s^{-1} 3 p{ }^{2} P_{1 / 2,3 / 2}$ levels in neutral sodium and its subsequent radiative decay back to the $3 s^{2} S_{1 / 2}$ ground level. Moreover, we later discuss the anisotropy parameter (angular distribution) and linear polarization of the fluorescence $\gamma_{2}$ photon as functions of incident photon energy, i.e., if the incident radiation is tuned over the $2 s^{-1} 3 p{ }^{2} P_{1 / 2,3 / 2}$ overlapping resonances. Finally, conclusions and a brief outlook of the present work are given in Sec. IV

Atomic units $\left(m_{e}=1, e=1, \hbar=1\right)$ are used throughout this paper unless stated otherwise.

\section{THEORY AND COMPUTATION}

We here consider the (combined) process

$$
\begin{aligned}
A\left(\alpha_{i} J_{i}\right)+\gamma_{1} & \longrightarrow\left\{\begin{array}{c}
A^{*}(\alpha J) \\
A^{*}\left(\alpha^{\prime} J^{\prime}\right)
\end{array}\right\} \\
& \longrightarrow A\left(\alpha_{f} J_{f}\right)+\gamma_{2}
\end{aligned}
$$

of the photoexcitation of an atom or ion and its subsequent fluorescence emission, which proceeds via two overlapping resonances. In contrast to the typical twostep model for the excitation and decay, we treat the whole process (11) together in order to allow for a coherence transfer during the excitation and decay of the atoms. In this process, the atom (or ion) is initially assumed to be in its ground level $\alpha_{i} J_{i}$ and is excited to some overlapping resonances $\alpha J$ and $\alpha^{\prime} J^{\prime}$ by absorbing the photon $\gamma_{1}$ with energy $\omega_{1}$. Owing to the finite lifetime of these resonances, which causes their overlap, they subsequently decay by photon emission $\gamma_{2}$ (with energy $\omega_{2}$ ) to some energetically lower-lying levels, say, $\alpha_{f} J_{f}$; cf. Fig. 1. While the J's here just denote the total angular momenta of the levels, the $\alpha^{\prime} s$ refer to all further quantum numbers that are needed for a unique specification of these levels. In process (1), the initial and final levels, $\alpha_{i} J_{i}$ and $\alpha_{f} J_{f}$, can both be the same, giving rise to the same photon energy $\omega_{1}=\omega_{2} \equiv \omega$ as we consider in our example below. Let us note here, moreover, that the "overlap" of the two resonances above is often caused by fast autoionization channels, and that the (second-step) fluorescence might be suppressed when compared to the ionization of the system.

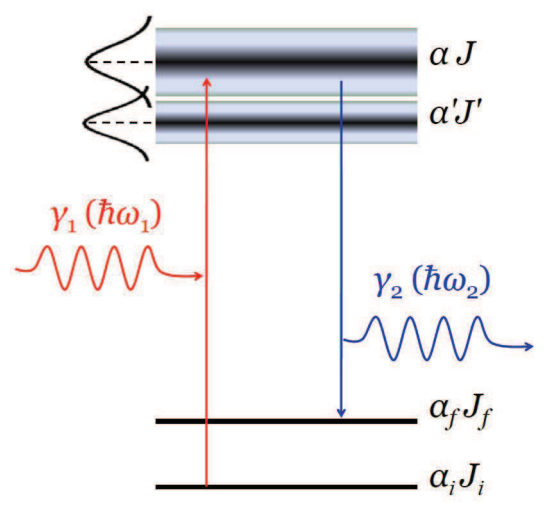

FIG. 1: (Color online) Level scheme for the (combined) process of the photoexcitation and subsequent fluorescence emission via two overlapping resonances. An atom (or ion) in the initial ground level $\alpha_{i} J_{i}$ absorbs the photon $\gamma_{1}$ and is excited to the overlapping $\alpha J$ and $\alpha^{\prime} J^{\prime}$ resonances, and subsequently decays to some low-lying levels $\alpha_{f} J_{f}$ via fluorescence emission of photon $\gamma_{2}$.

\section{A. Evaluation of transition amplitude}

The considered (two-step) process (1) of the photoexcitation and subsequent fluorescence emission of an atom or ion is quite analogue to the resonant Rayleigh scattering of photons by some atomic target. For the resonant excitation of atoms $\left(\omega=E_{\alpha J}-E_{\alpha_{i} J_{i}}\right.$ or $\omega=$ $E_{\alpha^{\prime} J^{\prime}}-E_{\alpha_{i} J_{i}}$ ), the (Rayleigh) scattering amplitude indeed contains singularities which can be removed by performing an infinite resummation of the radiative corrections for the resonant levels $\alpha J$ and $\alpha^{\prime} J^{\prime}$ [22, 23]. This resummation naturally leads to the occurrence of the linewidths in the denominators of the second-order transition amplitude. For the case of just two overlapping resonances $\alpha J$ and $\alpha^{\prime} J^{\prime}$, the scattering amplitude in the resonance approximation then takes the form 


$$
\begin{aligned}
\mathcal{M}_{M_{i}, M_{f}}^{\lambda_{1}, \lambda_{2}}(\omega)= & \sum_{M^{\prime}} \frac{\left\langle\alpha_{f} J_{f} M_{f}\left|\sum_{m} \boldsymbol{\alpha}_{m} \cdot \boldsymbol{\epsilon}_{\lambda_{2}}^{*} e^{-i \boldsymbol{k}_{2} \cdot \boldsymbol{r}_{m}}\right| \alpha^{\prime} J^{\prime} M^{\prime}\right\rangle\left\langle\alpha^{\prime} J^{\prime} M^{\prime}\left|\sum_{m} \boldsymbol{\alpha}_{m} \cdot \boldsymbol{\epsilon}_{\lambda_{1}} e^{i \boldsymbol{k}_{1} \cdot \boldsymbol{r}_{m}}\right| \alpha_{i} J_{i} M_{i}\right\rangle}{E_{\alpha_{i} J_{i}}-E_{\alpha^{\prime} J^{\prime}}+\omega+i \Gamma_{\alpha^{\prime} J^{\prime}} / 2} \\
& +\sum_{M} \frac{\left\langle\alpha_{f} J_{f} M_{f}\left|\sum_{m} \boldsymbol{\alpha}_{m} \cdot \boldsymbol{\epsilon}_{\lambda_{2}}^{*} e^{-i \boldsymbol{k}_{2} \cdot \boldsymbol{r}_{m}}\right| \alpha J M\right\rangle\left\langle\alpha J M\left|\sum_{m} \boldsymbol{\alpha}_{m} \cdot \boldsymbol{\epsilon}_{\lambda_{1}} e^{i \boldsymbol{k}_{1} \cdot \boldsymbol{r}_{m}}\right| \alpha_{i} J_{i} M_{i}\right\rangle}{E_{\alpha_{i} J_{i}}-E_{\alpha J}+\omega+i \Gamma_{\alpha J} / 2},
\end{aligned}
$$

where $\boldsymbol{k}_{1,2}$ and $\boldsymbol{\epsilon}_{\lambda_{1,2}}$ are the wave and polarization vectors of the photons $\gamma_{1}$ and $\gamma_{2}$, respectively. $\boldsymbol{r}_{m}$ and $\boldsymbol{\alpha}_{m}=$ $\left(\alpha_{x, m}, \alpha_{y, m}, \alpha_{z, m}\right)$ represent the coordinate and the vector of the Dirac matrices for the $m$ th electron. $\left|\alpha_{i} J_{i} M_{i}\right\rangle$ and $\left|\alpha_{f} J_{f} M_{f}\right\rangle$ characterize the initial and final states of the atom, while the $J$ 's and $M$ 's refer to the total angular momenta and their projection upon the z-axis. Moreover, $E_{\alpha J}-E_{\alpha_{i} J_{i}}$ and $\Gamma_{\alpha J}$ denote the excitation energy and natural linewidth of the resonance $\alpha J$, and analogue for the second "primed" resonance $\alpha^{\prime} J^{\prime}$. The operator $\sum_{m} \boldsymbol{\alpha}_{m} \cdot \boldsymbol{\epsilon}_{\lambda} e^{i \boldsymbol{k} \cdot \boldsymbol{r}_{m}}$ describes as usual the interaction of atomic electrons with the radiation field within the velocity gauge in terms of a sum of one-electron interaction operators. The second-order amplitude (2) can be further simplified if the operator $\boldsymbol{\alpha}_{\boldsymbol{m}} \cdot \boldsymbol{\epsilon}_{\lambda} e^{i \boldsymbol{k} \cdot \boldsymbol{r}_{m}}$ is decomposed into partial waves,

$$
\boldsymbol{\alpha}_{\boldsymbol{m}} \cdot \boldsymbol{\epsilon}_{\lambda} e^{i \boldsymbol{k} \cdot \boldsymbol{r}_{m}}=4 \pi \sum_{p L M_{L}} i^{L-p}\left[\boldsymbol{\epsilon}_{\lambda} \cdot \boldsymbol{Y}_{L M_{L}}^{(p) *}(\hat{\boldsymbol{k}})\right] \boldsymbol{\alpha}_{\boldsymbol{m}} \boldsymbol{a}_{L M_{L}}^{p}\left(\boldsymbol{r}_{\boldsymbol{m}}\right)
$$

where $\boldsymbol{Y}_{L M_{L}}^{(p)}(\hat{\boldsymbol{k}})$ is a vector spherical harmonics as function of $\hat{\boldsymbol{k}} \equiv \boldsymbol{k} /|\boldsymbol{k}|[24]$ and $\boldsymbol{a}_{L M_{L}}^{p}(\boldsymbol{r})$ represents the electric $(p=1)$ and magnetic $(p=0)$ multipole components of the radiation field. The explicit form of these components has been discussed at several places elsewhere in the literature [24].

In describing the process (1), we here choose the propagation direction $\hat{\boldsymbol{k}}_{1}$ of the incoming photon $\gamma_{1}$ as quantization axis (z-axis) and its polarization vector $\boldsymbol{\epsilon}_{\lambda_{1}}$ as $x$-axis. Then, the emitted fluorescence photon $\gamma_{2}$ is observed along some direction $\hat{\boldsymbol{k}}_{2}$ that is usually characterized by two angles $\hat{\boldsymbol{k}}_{2} \equiv(\theta, \varphi)$, the polar angle $\theta$ and the azimuthal angle $\varphi$ with regard to the $x z$ plane (cf. Fig. 2). For this choice of coordinates, the transition amplitude (2) can be written explicitly as

$$
\begin{aligned}
\mathcal{M}_{M_{i}, M_{f}}^{\lambda_{1}, \lambda_{2}}(\omega) & =\sum_{p_{1} L_{1} M_{L_{1}}} \sum_{p_{2} L_{2} M_{L_{2}}} i^{L_{1}-L_{2}}\left(i \lambda_{1}\right)^{p_{1}}\left(i \lambda_{2}\right)^{p_{2}}\left[L_{1}, L_{2}\right]^{1 / 2} \delta_{\lambda_{1} M_{L_{1}}} d_{M_{L_{2}} \lambda_{2}}^{L_{2}}(\theta) e^{-i M_{L_{2}} \varphi}(-1)^{J_{i}-J_{f}-M_{L_{1}}+1} \\
& \times\left\{\left(\sum_{M^{\prime}}\left\langle J_{f} M_{f}, L_{2} M_{L_{2}} \mid J^{\prime} M^{\prime}\right\rangle\left\langle J^{\prime} M^{\prime}, L_{1}-M_{L_{1}} \mid J_{i} M_{i}\right\rangle\right)\left[J^{\prime}, J_{i}\right]^{-1 / 2} \frac{\mathcal{T}_{p_{2} L_{2}}^{\prime} \mathcal{T}_{p_{1} L_{1}}^{\prime}}{E_{\alpha_{i} J_{i}}-E_{\alpha^{\prime} J^{\prime}}+\omega+i \Gamma_{\alpha^{\prime} J^{\prime}} / 2}\right. \\
& \left.+\left(\sum_{M}\left\langle J_{f} M_{f}, L_{2} M_{L_{2}} \mid J M\right\rangle\left\langle J M, L_{1}-M_{L_{1}} \mid J_{i} M_{i}\right\rangle\right)\left[J, J_{i}\right]^{-1 / 2} \frac{\mathcal{T}_{p_{2} L_{2}} \mathcal{T}_{p_{1} L_{1}}}{E_{\alpha_{i} J_{i}}-E_{\alpha J}+\omega+i \Gamma_{\alpha J} / 2}\right\} .
\end{aligned}
$$

Here, the short-hand notations $\mathcal{T}_{p_{1} L_{1}} \equiv\left\langle\alpha J\left\|\sum_{m} \boldsymbol{\alpha}_{m} \boldsymbol{a}_{L_{1}}^{p_{1}}\left(\boldsymbol{r}_{m}\right)\right\| \alpha_{i} J_{i}\right\rangle, \mathcal{T}_{p_{1} L_{1}}^{\prime} \equiv\left\langle\alpha^{\prime} J^{\prime}\left\|\sum_{m} \boldsymbol{\alpha}_{m} \boldsymbol{a}_{L_{1}}^{p_{1}}\left(\boldsymbol{r}_{m}\right)\right\| \alpha_{i} J_{i}\right\rangle$, and $\mathcal{T}_{p_{2} L_{2}} \equiv\left\langle\alpha_{f} J_{f}\left\|\sum_{m} \boldsymbol{\alpha}_{m} \boldsymbol{a}_{L_{2}}^{p_{2}}\left(\boldsymbol{r}_{m}\right)\right\| \alpha J\right\rangle, \mathcal{T}_{p_{2} L_{2}}^{\prime} \equiv\left\langle\alpha_{f} J_{f}\left\|\sum_{m} \boldsymbol{\alpha}_{m} \boldsymbol{a}_{L_{2}}^{p_{2}}\left(\boldsymbol{r}_{m}\right)\right\| \alpha^{\prime} J^{\prime}\right\rangle$ are used to denote the reduced transition amplitudes for the absorption of the exciting photon $\gamma_{1}$ and the emission of the fluorescence photon $\gamma_{2}$, respectively. Moreover, $[a, b] \equiv(2 a+1)(2 b+1)$, and the standard notations for the Wigner (small) $d$-function and the Clebsch-Gordan coefficients are employed.

\section{B. Density matrix of the fluorescence photon}

Since the transition amplitude (4) combines the excitation and the subsequent fluorescence emission, i.e. the photons $\gamma_{1}$ and $\gamma_{2}$, we can quite easily obtain the density matrix of the fluorescence photon $\gamma_{2}$ from these amplitudes [25, 26]. For the given choice of the coordinates, in particular, the density matrix of the photon $\gamma_{2}$ can be expressed in terms of (the helicity part of) the density matrix of the photon $\gamma_{1}$, sometimes called the helicity density matrix,

$$
\left\langle\hat{\boldsymbol{k}}_{2}, \lambda_{2}\left|\rho_{\gamma_{2}}\right| \hat{\boldsymbol{k}}_{2}, \lambda_{2}^{\prime}\right\rangle=\frac{1}{2 J_{i}+1} \sum_{M_{i}, M_{f}} \sum_{\lambda_{1} \lambda_{1}^{\prime}} \mathcal{M}_{M_{i}, M_{f}}^{\lambda_{1}, \lambda_{2}}(\omega) \mathcal{M}_{M_{i}, M_{f}}^{\lambda_{1}^{\prime}, \lambda_{2}^{\prime *}}(\omega)\left\langle\hat{\boldsymbol{k}}_{1}, \lambda_{1}\left|\rho_{\gamma_{1}}\right| \hat{\boldsymbol{k}}_{1}, \lambda_{1}^{\prime}\right\rangle .
$$

In deriving this formula, we have assumed that the atom is initially unpolarized and that its final state $\left|\alpha_{f} J_{f} M_{f}\right\rangle$ remains unobserved in the measurements. In the density matrix theory, moreover, the helicity density matrix of a 
photon is a $2 \times 2$ matrix that just describes polarization of the photon and, is usually parametrized by means of the three Stokes parameters [26, 27]

$$
\left\langle\hat{\boldsymbol{k}}, \lambda\left|\rho_{\gamma}\right| \hat{\boldsymbol{k}}, \lambda^{\prime}\right\rangle=\frac{1}{2}\left(\begin{array}{cc}
1+P_{3} & P_{1}-i P_{2} \\
P_{1}+i P_{2} & 1-P_{3}
\end{array}\right)
$$

Here, $P_{1,2}$ and $P_{3}$ characterize the linear and circular polarization of the photon, respectively. For unpolarized incident light $\left(P_{1}=P_{2}=P_{3}=0\right)$, which is the case that we are just considering in the present work, in addition, Eq. (5) can be further simplified to

$$
\left\langle\hat{\boldsymbol{k}}_{2}, \lambda_{2}\left|\rho_{\gamma_{2}}\right| \hat{\boldsymbol{k}}_{2}, \lambda_{2}^{\prime}\right\rangle=\frac{1}{2\left(2 J_{i}+1\right)} \sum_{M_{i}, M_{f}}\left(\mathcal{M}_{M_{i}, M_{f}}^{1, \lambda_{2}}(\omega) \mathcal{M}_{M_{i}, M_{f}}^{1, \lambda_{2}^{*}}(\omega)+\mathcal{M}_{M_{i}, M_{f}}^{-1, \lambda_{2}}(\omega) \mathcal{M}_{M_{i}, M_{f}}^{-1, \lambda_{2}^{\prime *}}(\omega)\right)
$$

Since both, the angular distribution and the (linear and circular) polarization of a photon are fully characterized by its density matrix [25 27], we are ready now to analyze and discuss these properties especially for the subsequent fluorescence emission, $\gamma_{2}$.

\section{Angular distribution and polarization parameters}

If, for example, the polarization of the fluorescence photon $\gamma_{2}$ remains unobserved, its angular distribution simply follows from the trace of the density matrix (7),

$$
\sigma\left(\hat{\boldsymbol{k}}_{2}\right)=\left\langle\hat{\boldsymbol{k}}_{2}, \lambda_{2}=+1\left|\rho_{\gamma_{2}}\right| \hat{\boldsymbol{k}}_{2}, \lambda_{2}^{\prime}=+1\right\rangle+\left\langle\hat{\boldsymbol{k}}_{2}, \lambda_{2}=-1\left|\rho_{\gamma_{2}}\right| \hat{\boldsymbol{k}}_{2}, \lambda_{2}^{\prime}=-1\right\rangle
$$

For an initially unpolarized atomic target and unpolarized incident photon $\gamma_{1}$, the angular distribution (8) of the $\gamma_{2}$ fluorescence light is azimuthally symmetric, thus independent of the angle $\varphi$, and can be characterized by just a single anisotropy parameter $\beta$ if the light is produced by an electric-dipole (E1) line emission [26],

$$
\sigma(\theta)=\frac{\sigma_{0}}{4 \pi}\left[1+\beta \mathrm{P}_{2}(\cos \theta)\right]
$$

It is necessary to mention that this expression is obtained within the E1 approximation. In expression (9), moreover, $\sigma_{0}$ denotes the total scattering cross section and $\mathrm{P}_{2}(\cos \theta)$ is the second-order Legendre polynomial as function of the polar angle $\theta$, taken with regard to the $z$ axis. Therefore, once we have the anisotropy parameter $\beta$, we also know the angular distribution of the fluorescence light $\gamma_{2}$.

Apart from the angular distribution, we can use the density matrix (7) to also derive the linear polarization of the fluorescence radiation. As usual in atomic and optical physics, the linear polarization is characterized by the two Stokes parameters $P_{1}$ and $P_{2}$ [26, 27]. For example, the parameter $P_{1}=\left(I_{0^{\circ}}-I_{90^{\circ}}\right) /\left(I_{0^{\circ}}+I_{90^{\circ}}\right)$ is just determined by the intensities of the fluorescence $\gamma_{2}$ light linearly polarized in parallel $\left(I_{0^{\circ}}\right)$ or perpendicular $\left(I_{90^{\circ}}\right)$ with regard to the plane spanned by the propagation direction of the $\gamma_{1}$ and $\gamma_{2}$ photons (cf. Fig. 2). Of course, as discussed above, this parameter $P_{1}$ can also be expressed in terms of the density matrix of the photon by using Eqs. (66)-(7),

$$
P_{1}\left(\hat{\boldsymbol{k}}_{2}\right)=\frac{\left\langle\hat{\boldsymbol{k}}_{2}, \lambda_{2}=+1\left|\rho_{\gamma_{2}}\right| \hat{\boldsymbol{k}}_{2}, \lambda_{2}^{\prime}=-1\right\rangle+\left\langle\hat{\boldsymbol{k}}_{2}, \lambda_{2}=-1\left|\rho_{\gamma_{2}}\right| \hat{\boldsymbol{k}}_{2}, \lambda_{2}^{\prime}=+1\right\rangle}{\left\langle\hat{\boldsymbol{k}}_{2}, \lambda_{2}=+1\left|\rho_{\gamma_{2}}\right| \hat{\boldsymbol{k}}_{2}, \lambda_{2}^{\prime}=+1\right\rangle+\left\langle\hat{\boldsymbol{k}}_{2}, \lambda_{2}=-1\left|\rho_{\gamma_{2}}\right| \hat{\boldsymbol{k}}_{2}, \lambda_{2}^{\prime}=-1\right\rangle} .
$$

With the use of the expressions (9) and (10), we can therefore readily explore the angular distribution and the linear polarization of the characteristic fluorescence light in the combined excitation and decay process (1). Moreover, the $P_{2}$ parameter is always zero for the case of unpolarized incident light as considered in this work.

\section{Computation of the reduced matrix elements}

It follows directly from above that any further analysis of the $\gamma_{2}$ angular distribution and polarization requires the computation of the second-order transition amplitude
(4) and, hence, the (usual) reduced matrix elements $\mathcal{T}_{p L}$ for single-photon bound-bound transitions [28, 29]. Since these reduced matrix elements occur very frequently in photoexcitation and radiative transition studies 30 32], they are readily available from different computer codes [33, 34], and not much need to be said about their de- 


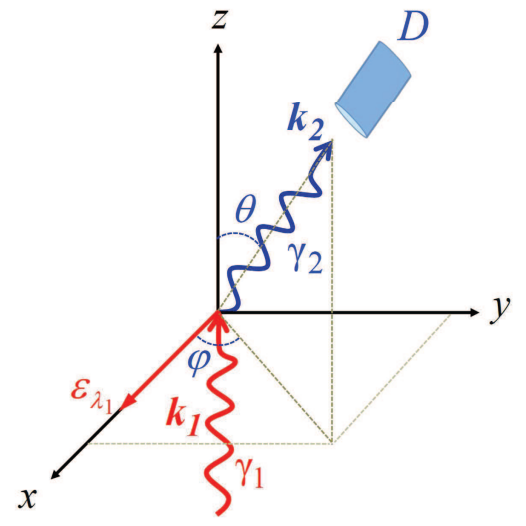

FIG. 2: (Color online) Geometry of the photoexcitation and subsequent radiative decay. The incident light $\gamma_{1}$ propagates along the $z$-axis (chosen as quantization axis) with its polarization vector $\epsilon_{\lambda_{1}}$ in the $x$-axis, while the fluorescence photon $\gamma_{2}$ is described by the two angles $(\theta, \varphi)$.

tailed computation. We here applied the multiconfiguration Dirac-Fock (MCDF) method 35] and especially the associated Grasp92/2K code [36, 37] to compute the energy levels and wave functions of all the relevant atomic states. In the MCDF method, an atomic state function (ASF) with well-defined parity $P$, total angular momentum $J$ and its component $M$, is approximated by a linear combination of a set of configuration state functions (CSF) with the same $P J M$,

$$
\psi_{\alpha}(P J M)=\sum_{r=1}^{n_{c}} c_{r}(\alpha)\left|\phi_{r}(P J M)\right\rangle .
$$

Here, $n_{c}$ denotes the number of CSF that are used in order to construct the $\mathrm{ASF}$ and, $c_{r}(\alpha)$ refers to the (so-called) configuration mixing coefficients. The CSF are constructed self-consistently on the basis of the Dirac-Coulomb Hamiltonian, while the relativistic and quantum-electrodynamical effects are incorporated into the coefficients $c_{r}(\alpha)$ by diagonalizing the matrix of the Dirac-Coulomb-Breit Hamiltonian in first-order perturbation theory [35-39]. Once these energy levels and wave functions are obtained, one can easily apply them to calculate all the required reduced matrix elements by using, for example, the RATIP code [33].

\section{RESULTS AND DISCUSSION}

\section{A. The $2 s \rightarrow 3 p$ photoexcitation and subsequent radiative decay of sodium atom}

Equations (2)-(7) are general and thus applicable to any atomic (or ionic) system with overlapping (excited) resonances, quite independent of the particular shell structure. As an example, we shall consider here the

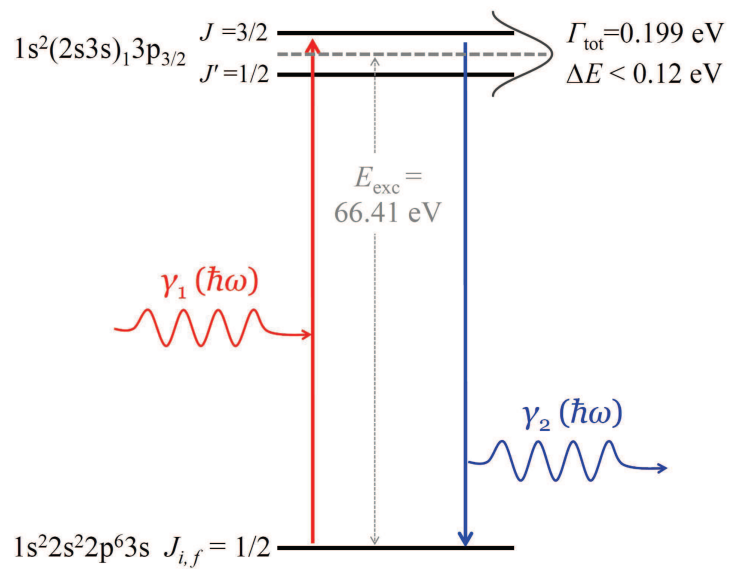

FIG. 3: (Color online) Level scheme of the $2 s \rightarrow 3 p$ innershell photoexcitation (red) and the subsequent radiative decay (blue) of atomic sodium. In the computations, we here use the experimentally known data for the total linewidth $\Gamma_{\text {tot }}$ and the central excitation energy $E_{\text {exc }}$ [43].

$2 s \rightarrow 3 p$ photoexcitation of an inner-shell electron and its subsequent fluorescence emission in atomic sodium,

$$
\begin{aligned}
& 1 s^{2} 2 s^{2} 2 p^{6} 3 s J_{i}=1 / 2+\gamma_{1} \\
& \longrightarrow\left\{\begin{array}{c}
\left(1 s^{2} 2 s 2 p^{6} 3 s\right)_{1} 3 p_{3 / 2} \\
\left(1 s^{2} 2 s 2 p^{6} 3 s\right)_{1} 3 p_{3 / 2} J^{\prime}=3 / 2
\end{array}\right\} \\
& \longrightarrow 1 s^{2} 2 s^{2} 2 p^{6} 3 s \quad J_{f}=1 / 2+\gamma_{2} .
\end{aligned}
$$

Note, that all the transitions are E1-allowed in this excitation and decay scheme (12), cf. Fig. 3. In the following, we can therefore restrict ourselves to the E1 approximation, i.e. to $p_{1}=p_{2}=1$, and $L_{1}=L_{2}=1$ in Eq. (4). In this approximation, just two reduced E1 matrix elements $\left\langle J_{f}=1 / 2\left\|\sum_{m} \boldsymbol{\alpha}_{m} \boldsymbol{a}_{1}^{1}\left(\boldsymbol{r}_{m}\right)\right\| J=1 / 2,3 / 2\right\rangle$ need to be calculated in order to obtain the transition amplitude (41), where we have omitted the electron configurations for the sake of brevity. The two $|J=1 / 2,3 / 2\rangle$ resonances overlap each other and cannot be resolved spectroscopically [40 43$]$. In the computations, we use the experimentally known data $0.199 \mathrm{eV}$ and $66.41 \mathrm{eV}$ for the total linewidth $\Gamma_{\text {tot }}$ and the central excitation energy $E_{\text {exc }}$ [43]. Moreover, since the $\left(1 s^{2} 2 s 2 p^{6} 3 s\right)_{1} 3 p_{3 / 2} \quad J=1 / 2,3 / 2$ overlapping resonances are well isolated from other excited levels of neutral sodium [44], we just 'tune' the resonances with the incident $\gamma_{1}$ light and omit all other excitations. In this particular example of sodium, the two resonances remain unresolved spectroscopically for the level splittings $|\Delta E| \equiv\left|E_{3 / 2}-E_{1 / 2}\right| \lesssim 0.12 \mathrm{eV}$ due to a resolution criteria for two overlapping resonances with (approximately) the same individual linewidths and the total linewidth $\Gamma_{\text {tot }} \simeq 0.2 \mathrm{eV}$. In our analysis below, therefore, we consider also level splittings $|\Delta E|$ which are smaller than the resolution criteria of $0.12 \mathrm{eV}$. For 
other splittings (much) larger than $0.12 \mathrm{eV}$, they are not physically significant since the yield of the subsequent fluorescence $\gamma_{2}$ photons is almost null when tuning photon energy of the incident $\gamma_{1}$ light between the two resonances. While the total linewidth of the overlapping resonances is experimentally known for neutral sodium, their individual linewidths are estimated to be the same and are expressed approximately as $\Gamma_{\alpha J}=\Gamma_{\alpha^{\prime} J^{\prime}} \simeq$ $\Gamma_{\text {tot }}\left(1-\Delta E^{2} / 2 \Gamma_{\text {tot }}^{2}\right)$ in terms of the total linewidth $\Gamma_{\text {tot }}$ and the (assumed) level splitting $\Delta E$.

Below, we shall apply Eqs. (2) -(10) in order to analyze the angular distribution and linear polarization of the $\gamma_{2}$ fluorescence emission following the $2 s \rightarrow 3 p$ photoexcitation of sodium via the $\left(1 s^{2} 2 s 2 p^{6} 3 s\right)_{1} 3 p_{3 / 2} \quad J=1 / 2,3 / 2$ overlapping resonances. In particular, we are interested how this fluorescence depends on both, the level sequence and the splitting of the $J=1 / 2,3 / 2$ resonances, if analyzed as a function of the photon energy of the incident light $\gamma_{1}$. In addition, we shall propose two independent scenarios for determining experimentally the sequence and splitting by measuring the angular distribution and linear polarization of the fluorescence light.

\section{B. Angular distribution of the fluorescence photons}

Let us start with the angular distribution of the $\gamma_{2} \mathrm{flu}-$ orescence emission from the two $\left(1 s^{2} 2 s 2 p^{6} 3 s\right)_{1} 3 p_{3 / 2} J=$ $1 / 2,3 / 2$ overlapping resonances after the photoexcitation. For different $\gamma_{1}$ photon energies of the incident light, the population of these levels is expected to differ

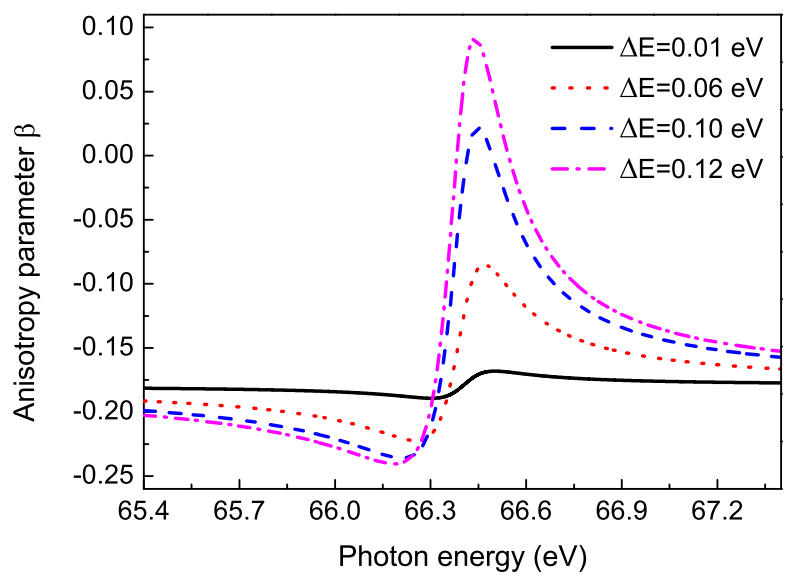

FIG. 4: (Color online) Anisotropy parameter $\beta$ for the angular distribution of the $\left(1 s^{2} 2 s 2 p^{6} 3 s\right)_{1} 3 p_{3 / 2} \quad J=1 / 2,3 / 2 \rightarrow$ $1 s^{2} 2 s^{2} 2 p^{6} 3 s \quad J_{f}=1 / 2$ fluorescence emission of sodium as functions of the photon energy $\omega$ of the incident $\gamma_{1}$ light. Results are shown for several assumed level splittings of the two $J=1 / 2,3 / 2$ overlapping resonances: $\triangle E=0.01 \mathrm{eV}$ (black solid line), $0.06 \mathrm{eV}$ (red dotted line), $0.10 \mathrm{eV}$ (blue dashed line), and $0.12 \mathrm{eV}$ (magenta dash-dotted line).

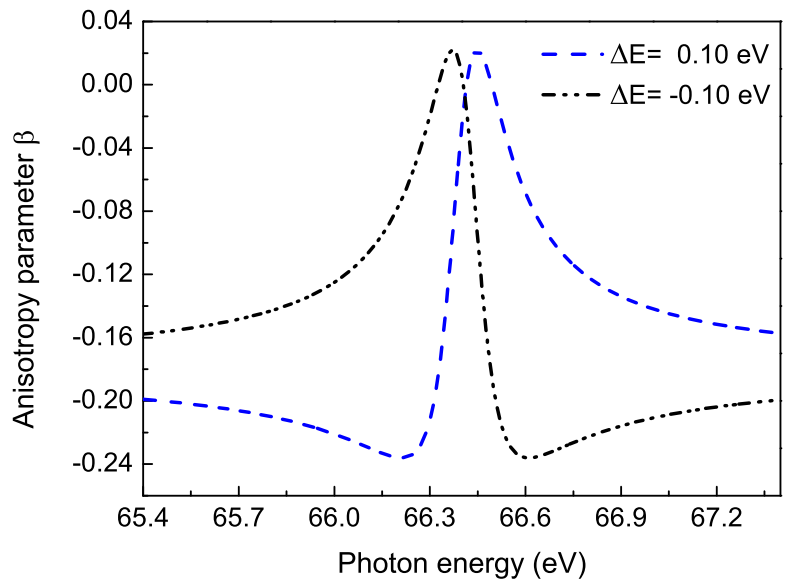

FIG. 5: (Color online) Anisotropy parameter $\beta$ for the angular distribution of the $\left(1 s^{2} 2 s 2 p^{6} 3 s\right)_{1} 3 p_{3 / 2} \quad J=1 / 2,3 / 2 \rightarrow$ $1 s^{2} 2 s^{2} 2 p^{6} 3 s \quad J_{f}=1 / 2$ fluorescence emission of sodium as functions of the photon energy $\omega$ of the incident $\gamma_{1}$ light. Results are shown for two assumed level splittings $\triangle E=0.10 \mathrm{eV}$ (blue dashed line) and $-0.10 \mathrm{eV}$ (black dash-dot-dotted line), which indicate that the (absolute) splitting of the two overlapping resonances remains the same but the sequence becomes opposite in both cases.

relative to each other and so also the angular distribution of the fluorescence emission. Moreover, the (coherent) excitation of the two resonances also depends on the level splitting [20, 21] and, this should thus become visible in the angular distribution as well.

Figure 4 displays the anisotropy parameter $\beta$ for the angular distribution of the $\left(1 s^{2} 2 s 2 p^{6} 3 s\right)_{1} 3 p_{3 / 2} \quad J=$ $1 / 2,3 / 2 \rightarrow 1 s^{2} 2 s^{2} 2 p^{6} 3 s \quad J_{f}=1 / 2$ fluorescence emission of sodium as functions of the photon energy $\omega$ of the incident light. Results are shown for different level splittings $|\Delta E| \equiv\left|E_{3 / 2}-E_{1 / 2}\right|=0.01,0.06,0.10$, and $0.12 \mathrm{eV}$ of the two overlapping resonances of sodium, which we assumed to be variable. As seen from this figure, the anisotropy parameter appears to be very sensitive for (almost) all level splittings with regard to the photon energy of the incident light. Moreover, this $\beta$ parameter also strongly depends upon the level splitting itself near the resonances. This latter dependence arises from the Lorentzian shape of the excitation distribution of the resonances due to their finite natural width.

Apart from the level splitting, the anisotropy parameter $\beta$ of the fluorescence light also depends on the level sequence if the photon energy of the incident light is tuned over the resonances. This can be seen from Fig. [5] where we plot the $\beta$ parameter as a function of the photon energy but for two opposite level splittings $\triangle E=0.10 \mathrm{eV}$ and $-0.10 \mathrm{eV}$, respectively. The opposite sign here indicates that the (absolute) splitting remains the same but that the level sequence has been reversed for the two overlapping resonances. For instance, the negative level 
splitting $\triangle E=-0.10 \mathrm{eV}$ means that the $J=3 / 2$ resonance lies lower than the $J=1 / 2$ one energetically by $0.10 \mathrm{eV}$. In particular, the shape of the two $\beta$ distributions occurs to be symmetric with regard to the central excitation energy of the overlapping resonances. This can be readily understood from formula (2) since the reversal of the level sequence is equivalent to the interchange in the $\gamma_{1}$ photon energy with regard to the central energy. This differs from the predicted angular distribution of the emitted fluorescence light in the two-step radiative cascade of $\mathrm{W}^{71+}$ ions, which was found insensitive with regard to the level sequence of the overlapping resonances due to the mutual cancelation of the sequence-dependent summation terms [20]. Therefore, accurate angular measurements of the fluorescence emission following the photoexcitation with sufficiently 'thin-banded' incident light might help identify both, the level sequence and splitting of closely-spaced energy levels in excited atoms or ions.

\section{Linear polarization of the fluorescence photons}

Until now, we just have discussed the angular distribution of the fluorescence emission from $2 s \rightarrow 3 p$ photoexcited sodium, if the photon energy of the incident light is tuned over the two $\left(1 s^{2} 2 s 2 p^{6} 3 s\right)_{1} 3 p_{3 / 2} \quad J=1 / 2,3 / 2$ overlapping resonances at about $66.4 \mathrm{eV}$. Alternatively, we may consider and analyze also the linear polarization of this fluorescence light, which can be measured nowadays with quite high accuracy either by means of solid-state Compton polarimeters [45, 46] or, even more precisely, with Bragg crystal polarimeters [47 49].

In Fig. 6. we therefore display the linear polarization of the fluorescence light $\gamma_{2}$ that is emitted perpendicu$\operatorname{lar}\left(\theta=90^{\circ}\right)$ to the propagation direction of the inci-

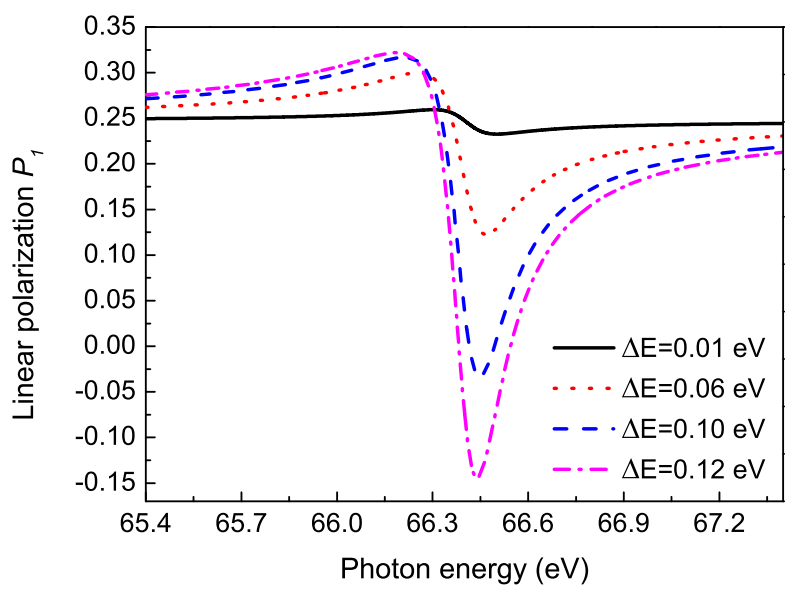

FIG. 6: (Color online) The same as Fig. 4 but for the linear polarization of the fluorescence $\gamma_{2}$ light emitted perpendicular $\left(\theta=90^{\circ}\right)$ to the propagation direction of the incident $\gamma_{1}$ light.

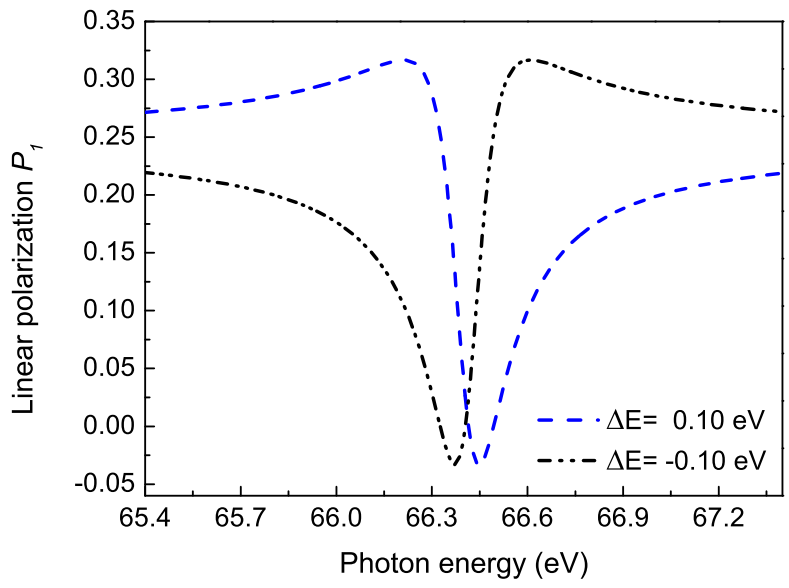

FIG. 7: (Color online) The same as Fig. 5 but for the linear polarization of the fluorescence $\gamma_{2}$ light emitted perpendicular $\left(\theta=90^{\circ}\right)$ to the propagation direction of the incident $\gamma_{1}$ light.

dent light $\gamma_{1}$. Again, the degree of linear polarization is shown as functions of the frequency of the incident light and for the same (assumed) level splittings of the two $\left(1 s^{2} 2 s 2 p^{6} 3 s\right)_{1} 3 p_{3 / 2} \quad J=1 / 2,3 / 2$ overlapping resonances. Similar as for the angular distribution, the degree of linear polarization depends on the level sequence and splitting, and here even at a larger absolute scale and with a change of its sign near to the central transition frequency. In addition, Fig. 7 displays the linear polarization for a level splitting of $0.1 \mathrm{eV}$ and its symmetry with regard to the central frequency if the level sequence is interchanged. As seen from these figures, accurate polarization measurements could also serve as an alternative and independent route to identify the sequence and splitting of overlapping resonances. Finally, we have to mention here that the incident $\gamma_{1}$ light is assumed to be monochromatic in the angular and polarization analysis of the fluorescence photon above. The use of a non-monochromatic incident light could weaken (more or less) the obtained angular and polarization dependence upon the (central) energy of the light, depending on the linewidth of the laser used. Nevertheless, this dependence still remains strong enough to be observable by using the present-day photon detectors.

\section{SUMMARY AND OUTLOOK}

In summary, the photoexcitation and subsequent fluorescence emission of atoms have been studied within the framework of the density matrix and second-order perturbation theory. Attention has been paid especially to the angular distribution and the linear polarization of the fluorescence as observed from (partially) overlapping resonances and how such measurements would be affected by the level sequence and splitting of the 
resonances involved. Detailed MCDF calculations were performed for the $1 s^{2} 2 s^{2} 2 p^{6} 3 s \quad J_{i}=1 / 2+\gamma_{1} \rightarrow$ $\left(1 s^{2} 2 s 2 p^{6} 3 s\right)_{1} 3 p_{3 / 2} J=1 / 2,3 / 2 \rightarrow 1 s^{2} 2 s^{2} 2 p^{6} 3 s J_{f}=$ $1 / 2+\gamma_{2}$ photoexcitation and subsequent photon emission of sodium atoms. It is predicted that the angular distribution and linear polarization of the $\gamma_{2}$ fluorescence photons strongly depend upon the level sequence and splitting of the $\left(1 s^{2} 2 s 2 p^{6} 3 s\right)_{1} 3 p_{3 / 2} \quad J=1 / 2,3 / 2$ resonances, if analyzed as functions of the frequency of the incident light. This dependence is caused by the non-negligible linewidth of the (overlapping) resonances which lead to a coherence transfer in the population of the resonances. This coherence transfer also affects the angular and polarization properties of the emitted fluorescence light. We therefore suggest that accurate measurements of the angular distribution and linear polarization of fluorescence light can be utilized to help identify the sequence and splitting of closely-spaced atomic (or ionic) energy levels, even if these levels cannot be resolved spectroscopically.

With the recent progress of light sources and photon detection techniques, the proposed measurements are feasible today. For example, laser-induced fluorescence spectroscopy or synchrotron radiation can be utilized for such energy-selective measurements of the subsequent fluorescence emission. In addition, the change of the obtained anisotropy parameter and linear polarization is large enough as functions of the incident photon energy to be measured by using present-day photon detector and polarimeter.

\section{Acknowledgments}

Z.W.W. acknowledges the support from the Helmholtz Institute Jena and the Research School of Advanced Photon Science of Germany. This work has been supported by the BMBF under Contract No. 05K13VHA and by the National Natural Science Foundation of China under Grant Nos. 11464042, 11274254, and U1332206.
[1] I. Martinson and A. Gaupp, Phys. Rep. 15, 113 (1974).

[2] B. Sonntag and P. Zimmermann, Rep. Prog. Phys. 55, 911 (1992).

[3] M. Vogel and W. Quint, Phys. Rep. 490, 1 (2010).

[4] S. Hell and E. H. K. Stelzer, Opt. Commun. 93, 277 (1992).

[5] W. F. Mueller, J. A. Church, T. Glasmacher, D. Gutknecht, G. Hackmana, P. G. Hansen, Z. Hu, K. L. Miller, and P. Quirin, Nucl. Instr. Meth. Phys. Res. A 466, 492 (2001).

[6] S. Huotari, Gy. Vankó, F. Albergamo, C. Ponchut, H. Graafsma, C. Henriquet, R. Verbeni, and G. Monaco, J. Synchrotron Rad. 12, 467 (2005).

[7] Z. W. Wu, J. Jiang, and C. Z. Dong, Phys. Rev. A 84, 032713 (2011).

[8] Z. W. Wu, C. Z. Dong, and J. Jiang, Phys. Rev. A 86, $022712(2012)$.

[9] S. Fritzsche, A. Surzhykov, and Th. Stöhlker, Phys. Rev. Lett. 103, 113001 (2009).

[10] S. Fritzsche, A. Surzhykov, A. Gumberidze, and T. Stöhlker, New J. Phys. 14, 083018 (2012).

[11] Z. M. Hu, X. Y. Han, Y. M. Li, D. Kato, X. M. Tong, and N. Nakamura, Phys. Rev. Lett. 108, 073002 (2012).

[12] H. Jörg, Z. Hu, H. Bekker, M. A. Blessenohl, D. Hollain, S. Fritzsche, A. Surzhykov, J. R. Crespo López-Urrutia, and S. Tashenov, Phys. Rev. A 91, 042705 (2015).

[13] A. Surzhykov, Y. Litvinov, T. Stöhlker, and S. Fritzsche, Phys. Rev. A 87, 052507 (2013).

[14] Z. W. Wu, A. Surzhykov, and S. Fritzsche, Phys. Rev. A 89, 022513 (2014).

[15] A. Surzhykov, S. Fritzsche, A. Gumberidze, and T. Stöhlker, Phys. Rev. Lett. 88, 153001 (2002).

[16] G. Weber, H. Bräuning, A. Surzhykov, C. Brandau, S. Fritzsche, S. Geyer, S. Hagmann, S. Hess, C. Kozhuharov, R. Märtin, N. Petridis, R. Reuschl, U. Spillmann, S. Trotsenko, D. F. A. Winters, and Th. Stöhlker, Phys. Rev. Lett. 105, 243002 (2010).
[17] Y. Shimizu, H. Yoshida, K. Okada, Y. Muramatsu, N. Saito, H. Okashi, Y. Tamenori, S. Fritzsche, N. M. Kabachnik, H. Tanaka, and K. Ueda, J. Phys. B 33, L685 (2000).

[18] K. Ueda, Y. Shimizu, H. Chiba, M. Kitajima, H. Tanaka, S. Fritzsche, and N. M. Kabachnik, J. Phys. B 34, 107 (2001).

[19] M. Kitajima, M. Okamoto, Y. Shimizu, H. Chiba, S. Fritzsche, N. M. Kabachnik, I. P. Sazhina, F. Koike, T. Hayaishi, H. Tanaka, Y. Sato, and K. Ueda, J. Phys. B 34, 3829 (2001).

[20] Z. W. Wu, N. M. Kabachnik, A. Surzhykov, C. Z. Dong, and S. Fritzsche, Phys. Rev. A 90, 052515 (2014).

[21] Z. W. Wu, A. Surzhykov, N. M. Kabachnik, C. Z. Dong, and S. Fritzsche, J. Phys.: Conf. Ser. 635, 012020 (2015).

[22] V. M. Shabaev, Phys. Rep. 356, 119 (2002).

[23] O. Yu. Andreev, L. N. Labzowsky, G. Plunien, and D. A. Solovyev, Phys. Rep. 455, 135 (2008).

[24] D. A. Varshalovich, A. N. Moskalev, and V. K. Khersonskii, Quantum Theory of Angular Momentum (World Scientific, Singapore, 1988).

[25] N. M. Kabachnik, S. Fritzsche, A. N. Grum-Grzhimailo, M. Meyer, and K. Ueda, Phys. Rep. 451, 155 (2007).

[26] V. V. Balashov, A. N. Grum-Grzhimailo, and N. M. Kabachnik, Polarization and Correlation Phenomena in Atomic collisions (Kluwer Academic, New York, 2000).

[27] K. Blum, Density Matrix Theory and Applications (Plenum, New York, 1981).

[28] A. Surzhykov, U. D. Jentschura, Th. Stöhlker, and S. Fritzsche, Phys. Rev. A 73, 032716 (2006).

[29] S. Fritzsche, N. M. Kabachnik, and A. Surzhykov, Phys. Rev. A 78, 032703 (2008).

[30] I. P. Grant, Adv. Phys. 19, 747 (1970); J. Phys. B 7, 1458 (1974).

[31] S. Fritzsche, A. Surzhykov, and Th. Stöhlker, Phys. Rev. A 72, 012704 (2005).

[32] M. K. Inal, A. Surzhykov, and S. Fritzsche, Phys. Rev. 
A 72, 042720 (2005).

[33] S. Fritzsche, Comput. Phys. Commun. 183, 1525 (2012).

[34] M. F. Gu, Can. J. Phys. 86, 675 (2008).

[35] I. P. Grant, Relativistic Quantum Theory of Atoms and Molecules: Theory and Computation (Springer, New York, 2007).

[36] F. A. Parpia, C. F. Fischer, and I. P. Grant, Comput. Phys. Commun. 94, 249 (1996).

[37] P. Jönsson, X. He, C. F. Fischer, and I. P. Grant, Comput. Phys. Commun. 177, 597 (2007)

[38] P. Indelicato and J. P. Desclaux, Phys. Rev. A 42, 5139 (1990).

[39] S. Fritzsche, C. F. Fischer, and G. Gaigalas, Comput. Phys. Commun. 148, 103 (2002).

[40] R. E. LaVilla, G. Mehlman, and E. B. Saloman, J. Phys. B 14, L1 (1981).

[41] L. Journel, B. Rouvellou, D. Cubaynes, J. M. Bizau, F. J. Wuilleumier, M. Richter, P. Sladeczek, K.-H. Selbmann, and P. Zimmermann, J. Physique IV. 3, C6-217 (1993).

[42] P. N. Juranic, J. Nordberg, and R. Wehlitz, Phys. Rev. A 74, 042707 (2006)

[43] T. Osawa, Y. Tohyama, S. Obara, T. Nagata, Y. Azuma, and F. Koike, J. Phys. B 41, 245206 (2008).

[44] NIST Atomic Spectra Database Levels Form, Version 5, //physics.nist.gov/PhysRefData/ASD/levels_form.html

[45] S. Tashenov, Th. Stöhlker, D. Banaś, K. Beckert, P. Beller, H. F. Beyer, F. Bosch, S. Fritzsche, A. Gumberidze, S. Hagmann, C. Kozhuharov, T. Krings, D. Liesen, F. Nolden, D. Protic, D. Sierpowski, U. Spillmann, M. Steck, and A. Surzhykov, Phys. Rev. Lett. 97, 223202 (2006).

[46] S. Tashenov, T. Bäck, R. Barday, B. Cederwall, J. Enders, A. Khaplanov, Yu. Poltoratska, K.-U. Schässburger, and A. Surzhykov, Phys. Rev. Lett. 107, 173201 (2011).

[47] P. Beiersdorfer, D. A. Vogel, K. J. Reed, V. Decaux, J. H. Scofield, K. Widmann, G. Hölzer, E. Förster, O. Wehrhan, D. W. Savin, and L. Schweikhard, Phys. Rev. A 53, 3974 (1996).

[48] N. Nakamura, D. Kato, N. Miura, T. Nakahara, and S. Ohtani, Phys. Rev. A 63, 024501 (2001).

[49] T. Kämpfer, I. Uschmann, Z. W. Wu, A. Surzhykov, S. Fritzsche, E. Förster, and G. G. Paulus, Phys. Rev. A 93, 033409 (2016). 